\title{
Spatial thin-walled reinforced concrete structures taking into account physical nonlinearity in SCAD software. Rod finite element
}

\author{
Sergiy Fialko ${ }^{1}$, Viktor Karpilowskyi ${ }^{2}$ \\ ${ }^{I}$ Faculty of Physics, Mathematics and Computer Science, Cracow University of Technology, Kraków, Poland \\ ${ }^{2}$ IT Company Scad Soft, Kiev, Ukraine \\ E-mail: ${ }^{1}$ sergiy.fialko@gmail.com (corresponding author)
}

\begin{abstract}
This paper considers a spatial frame bar finite element for modeling reinforced concrete beams and columns. Both concrete and reinforcement are described by the equations of the deformation theory of plasticity and the theory of plastic flow. Degradation of concrete during cracking is modeled by the descending branch of the $\sigma-\varepsilon$ diagram (the deformation theory of plasticity), as well as the compression of the yield surface and its displacement in the space of principal stresses (the plastic flow theory). The longitudinal reinforcement is considered discretely. It is assumed that there is no reinforcement slipping in concrete. The paper provides the results of the studies that reveal the causes of computational instability related to the presence of a descending branch of the $\sigma-\varepsilon$ diagram for concrete, and proposes ways to overcome it. The reliability of the obtained results is confirmed by comparing them with the results of experimental studies performed by other researchers, as well as with the results of numerical solutions obtained by the particle method. This paper also provides an example of the nonlinear analysis of the fragment of a multi-storey building from the SCAD Soft collection of problems (www.scadsoft.com).
\end{abstract}

Keywords: finite element method, reinforced concrete, nonlinear analysis, plasticity, softening.

\section{Introduction}

Many FEA software packages, such as (ABAQUS, 2012), (ANSYS, 1999), (DIANA, 2014) and others, provide the user with a finite element library, material models and nonlinear analysis methods for modeling the behavior of reinforced concrete structures taking into account the elasto-plastic behavior of both concrete and reinforcement, as well as cracking in concrete. Nevertheless, the vast majority of calculations today are based on a linear elastic material model using finite elements that do not contain any information about the reinforcement. In other words, the rigidity of these elements depends neither on the diameter of reinforcement nor on its spacing. Internal forces are determined and the reinforcement is selected on the basis of such a calculation. And the fact that the presence of reinforcement changes the rigidities of structural elements compared to those assumed in the calculation, which in turn can lead to a different distribution of forces (it is typical for statically indeterminate systems) is completely ignored. The obvious fact that concrete is a physically nonlinear material, and its $\sigma-\varepsilon$ diagram has almost no linear segments is ignored as well.

At the same time, there are a large number of scientific researches proposing different models of concrete (e.g. Jouan, Kotronis, \& Collin, 2014), (e.g. Sukumar, Moes, Moran, \& Belytschko, 2000; Wang, 2013; Jirásek \& Bažant, 2002; Belytschko \& Rabczuk, 2006) and others. However, the proposed approaches are so complex, that they are applied only to an individual beam, individual slab, individual plane frame etc.

It is known that the behavior of an individual beam, column, floor slab, etc., may differ significantly from the case when they are considered together as a part of a structure. On the other hand, it is too expensive to perform a nonlinear analysis for the entire structure, and in many cases unnecessary. Therefore, we suggest to produce a traditional linear calculation of the entire structure and assign the diameters and step values between rods of reinforcement. Then select the most loaded fragments of this model, apply the reactions of the removed constraints and the most dangerous load to each of the selected fragments, and perform the nonlinear analysis.

It all seems paradoxical, so the developers of SCAD (e.g. Karpilovskyi et al., 2018) have added the following finite elements to the finite element library: four-node and three-node isoparametric finite elements for the analysis of medium-thickness plates (Bathe, 1996) and shells (Fialko \& Karpilovskyi, 2017; Fialko, 2015, 2018), and a spatial frame finite element, which will be considered in this paper.

(C) 2019 Authors. Published by VGTU Press. This is an open-access article distributed under the terms of the Creative Commons Attribution (http://creativecommons.org/licenses/by/4.0/) License, which permits unrestricted use, distribution, and reproduction in any medium, provided the original author and source are credited. 


\section{Problem formulation}

The behavior of concrete is described both by the relations of the deformation theory of plasticity (CM1), (Bezukhov, 1961; Ilyushin, 1948), formulated in terms of increments (Fialko, 2018), and by the plastic flow theory using the Drucker-Prager yield criterion (Criesfield, V. 2, 2000) (CM2) or the surface proposed in (e.g. Geniev, Kissyuk, \& Tyupin, 1974) as a strength surface and used as a yield surface (CM3). Hereinafter CM stands for constitutive model. Degradation of concrete during cracking is modeled by the descending branch of the $\sigma-\varepsilon$ diagram in the case of the deformation theory of plasticity (CM1), as well as the compression of the yield surface (isotropic softening) and its displacement in the space of principal stresses (kinematic softening) in the case of the plastic flow theory (CM1, CM2). In the case of the deformation theory of plasticity, the $\sigma-\varepsilon$ diagram proposed by the European Commission on concrete is used in the tension area, and a trilinear diagram is used in the tension area (e.g. Bathe, Walczak, Welch, \& Mistry, 1989) (Figure 1). In the case of the plastic flow theory, the compression of the yield surface is determined by the $\sigma-\varepsilon_{\text {ps }}$ diagram shown in Figure 2.

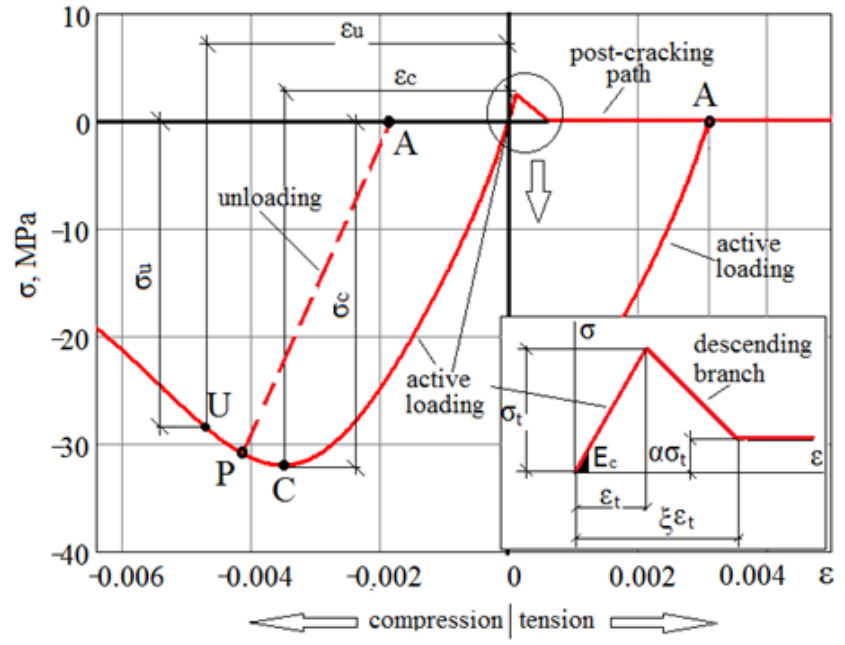

Figure 1. The $\sigma-\varepsilon$ diagram for concrete. Deformation theory of plasticity

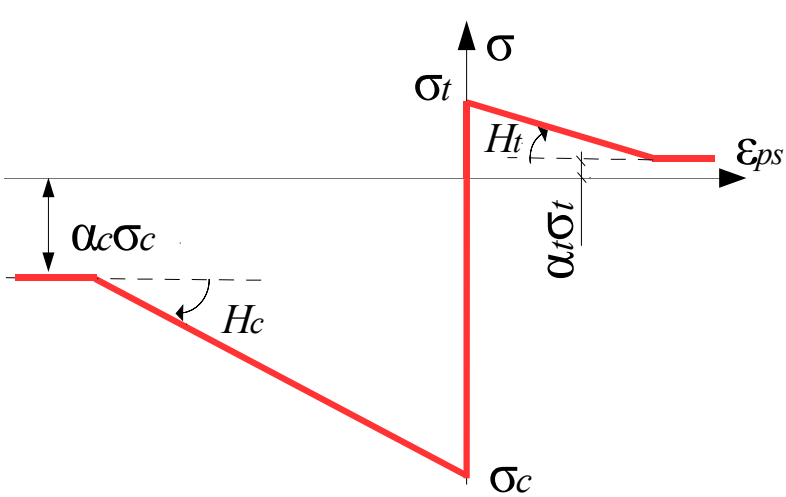

Figure 2. The $\sigma-\varepsilon$ diagram for concrete. Plastic flow theory

Here $\varepsilon_{\mathrm{ps}}$ is the plastic strain measure, $\sigma_{\mathrm{t}}, \sigma_{\mathrm{c}}$ are ultimate tensile and compressive strength of concrete respectively, $\varepsilon_{\mathrm{t}}, \varepsilon_{\mathrm{c}}$ are ultimate tensile and compressive strains respectively, $\sigma_{\mathrm{u}}$ is the limit compressive stress in concrete, $\xi$ is the parameter defining the length of the descending branch in the tension area, $\alpha$ is the parameter defining the remaining strength of concrete during cracking (usually $\alpha=0), H_{t}=E_{t} /\left(1-E_{t} / E\right.$ ), $E_{t}$ is the softening modulus of concrete in the tension area, $\mathrm{E}$ is the initial elastic modulus of concrete, $\mathrm{t} \leftrightarrow \mathrm{c}$.

In the CM1 case point $\mathrm{C}$ corresponds to the ultimate compressive strength of concrete (Figure 1), point $U-$ to the limit strain of concrete in the compression area, point $\mathrm{P}$ - to the start of unloading, point $\mathrm{A}-$ to the transition from the compression area to the tension area and vice versa. The residual strains are calculated, the origin on the strain axis is transferred to the point $\mathrm{A}$ and active loading begins in the area where the image point was after passing the point A. If the image point is in the tension area, and the concrete has already cracked during the previous loading history, then the image point slides along the strain axis $(\sigma=0)$ or parallel to the strain axis $\left(\sigma=\alpha \sigma_{t}\right)$.

In the CM2 and CM3 case, the concrete has elastic behavior in accordance with Hooke's law inside the body bounded by the yield surface. When the Drucker-Prager yield criterion is used, the yield surface is given by the following equation

$$
f=D I_{1}+\sqrt{J_{2}}-\sigma_{0}
$$

where $D=\frac{\bar{\sigma}_{c}-\bar{\sigma}_{t}}{\sqrt{3}\left(\bar{\sigma}_{c}+\bar{\sigma}_{t}\right)}, \sigma_{0}=\frac{\bar{\sigma}_{c} \bar{\sigma}_{t}}{2 \sqrt{3}\left(\bar{\sigma}_{c}+\bar{\sigma}_{t}\right)}, I_{1}$ is the first invariant of the stress tensor, $J_{2}$ is the second invariant of the stress deviator. When the CM3 model is used, the yield surface is represented as

$$
f=3 \bar{\alpha}^{2} b I_{1}+\frac{3 \sqrt{3}}{2} \beta\left(a-b I_{1}\right) J_{3} J_{2}^{-\frac{3}{2}}+3 J_{2}-\sigma_{0},
$$


where $a=\bar{\sigma}_{c} \bar{\sigma}_{t}, b=\bar{\sigma}_{c}+\bar{\sigma}_{t}, \bar{\alpha} \in(0.531, \sqrt{3}]$ is the parameter defining the deviation of paraboloid from the circular shape, $\beta=1-3 \bar{\alpha}^{2}, \sigma_{0}=3 a \bar{\alpha}^{2}, J_{3}=\operatorname{det}\left(D_{\sigma}\right), D_{\sigma}$ is the stress deviator.

After the image point reaches the yield surface (plastic flow), the compressive and tensile strength of concrete change as follows:

$$
\bar{\sigma}_{t}=\left\{\begin{array}{l}
\sigma_{t}+H_{t} \varepsilon_{p s}, \quad \sigma_{t}+H_{t} \varepsilon_{p s}>\alpha \sigma_{t} \\
\alpha \sigma_{t}, \quad \sigma_{t}+H_{t} \varepsilon_{p s} \leq \alpha \sigma_{t}
\end{array}, t \leftrightarrow c .\right.
$$

Therefore, in the case of the plastic flow, the yield surface is compressed and moves in the space of the principal stresses in accordance with the descending branches $\left(\mathrm{H}_{\mathrm{t}}<0, \mathrm{t} \leftrightarrow \mathrm{c}\right)$ of the $\sigma-\varepsilon_{\mathrm{ps}}$ diagram (Figure 2).

The anisotropy of concrete is neglected compared to the structural anisotropy introduced by reinforcement.

The behavior of reinforcement is described both by the relations of the deformation theory of plasticity formulated in terms of residual strains (CM1), and by the theory of plastic flow using the von Mises yield criterion (CM2 and CM3). A symmetric bilinear $\sigma-\varepsilon$ diagram is assumed. Shear stiffness of reinforcement is taken into account in addition to its axial stiffness. The longitudinal reinforcement is considered discretely.

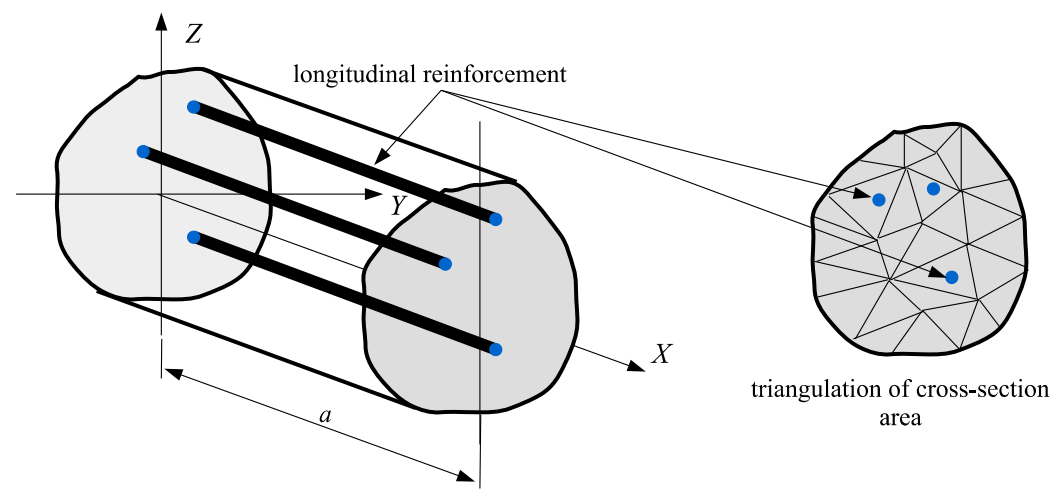

Figure 3. Spatial frame finite element with longitudinal reinforcement

S.P. Timoshenko shear model is used. A bar cross-section can have arbitrary shape with any number of holes and cuts. Numerical integration is used to calculate the integrals over the bar volume, therefore the cross-section of the bar is meshed into triangles (Figure 3), and longitudinal reinforcement bars are not snapped to mesh nodes. The kinematic hypothesis of a non-deformable cross-section is used to reduce a three-dimensional problem to a onedimensional one. In addition, the classic static hypothesis is used, according to which $\sigma_{\mathrm{y}}=\sigma_{\mathrm{z}}=\tau_{\mathrm{yz}}=0$. The longitudinal axis s of a rebar is parallel to the longitudinal axis OX (Figure 3), and the $\mathrm{n}$ and $\mathrm{z}$ axes are parallel to the $\mathrm{OY}$ and $\mathrm{OZ}$ axes respectively. As a result of these hypotheses the stress and strain tensors, $\boldsymbol{\sigma}$ and $\boldsymbol{\varepsilon}$, for concrete, and $\boldsymbol{\sigma}_{\mathrm{s}}$ and $\boldsymbol{\varepsilon}_{\mathrm{s}}$ for a rebar take the following form:

$$
\boldsymbol{\varepsilon}=\left(\begin{array}{ccc}
\varepsilon_{x} & 0.5 \gamma_{x y} & 0.5 \gamma_{x z} \\
0.5 \gamma_{y x} & -v \varepsilon_{x} & 0 \\
0.5 \gamma_{z x} & 0 & -v \varepsilon_{x}
\end{array}\right), \quad \boldsymbol{\sigma}=\left(\begin{array}{ccc}
\sigma_{x} & \tau_{x y} & \tau_{x z} \\
\tau_{y x} & 0 & 0 \\
\tau_{z x} & 0 & 0
\end{array}\right), \quad \boldsymbol{\varepsilon}_{s}=\left(\begin{array}{ccc}
\varepsilon_{s} & 0.5 \gamma_{s n} & 0.5 \gamma_{s z} \\
0.5 \gamma_{n s} & -v_{s} \varepsilon_{s} & 0 \\
0.5 \gamma_{z s} & 0 & -v_{s} \varepsilon_{s}
\end{array}\right), \quad \boldsymbol{\sigma}_{s}=\left(\begin{array}{ccc}
\sigma_{s} & \tau_{s n} & \tau_{s z} \\
\tau_{n s} & 0 & 0 \\
\tau_{z s} & 0 & 0
\end{array}\right),
$$

where $v$ is the Poisson's ratio for concrete, and $v_{\mathrm{s}}$ is the Poisson's ratio for reinforcement. It is assumed that concrete and reinforcement work together (with no sliding):

$$
\varepsilon_{s}=\varepsilon_{x}, \gamma_{s n}=\gamma_{x y}, \gamma_{s z}=\gamma_{x z} .
$$

The principle of virtual work is used to obtain the governing relations:

$$
\int_{V} \boldsymbol{\sigma}: \delta \boldsymbol{\varepsilon} d V+\sum_{s} A_{s} \int_{0}^{a} \boldsymbol{\sigma}_{s}: \delta \boldsymbol{\varepsilon}_{s} d x-\delta A_{e x t}=0,
$$

where $V$ is the volume of a finite element, $A_{\mathrm{s}}$ is the cross-sectional area of the s-th rebar, a is the length, $\delta A_{\text {ext }}$ is the virtual work of external forces. Summation is performed for all longitudinal reinforcement bars. Linear shape functions are used: $N_{1}(x)=1-x / a, N_{2}(x)=x / a$. In order to overcome the shear locking, shear strains are given as $\gamma_{x y}(x, z)=0.5\left(\gamma_{x y}(0, z)+\gamma_{x y}(a, z)\right), y \leftrightarrow z$. Strains are related to nodal displacements for concrete as follows: $\vec{\varepsilon}=\boldsymbol{\Phi}(x, y, z) \mathbf{q}_{e}$, where $\vec{\varepsilon}$ is a vector composed of strain tensor components $\boldsymbol{\varepsilon}$, $\boldsymbol{\Phi}$ is the deformation matrix, 
$\mathbf{q}_{e}=\left\{\begin{array}{llllllllllllll}U_{i} & V_{i} & W_{i} & \theta_{i}^{x} & B_{i}^{y} & B_{i}^{z} & U_{j} & V_{j} & W_{j} & \theta_{j}^{x} & B_{j}^{y} & B_{j}^{z}\end{array}\right\}_{e}$ is the vector of nodal displacements of a finite element e in the local coordinate system, $\mathrm{U}, \mathrm{V}, \mathrm{W}$ are the translational displacements in the direction of the OX, OY, $\mathrm{OZ}$ axes, respectively, $\theta^{\mathrm{x}}, \theta^{\mathrm{y}}$. $\theta^{\mathrm{z}}$. are the rotation angles, $\mathrm{B}^{\mathrm{y}}=-\theta^{\mathrm{z}}, \mathrm{B}^{\mathrm{z}}=\theta^{\mathrm{y}}, \mathrm{i}, \mathrm{j}$ are the finite element node numbers. The deformation matrix for concrete has the form:

$$
\boldsymbol{\Phi}(x, y, z)=\left(\begin{array}{ccccccccccccc}
-\frac{1}{a} & 0 & 0 & 0 & -\frac{y}{a} & -\frac{z}{a} & \frac{1}{a} & 0 & 0 & 0 & \frac{y}{a} & \frac{z}{a} \\
0 & -\frac{1}{a} & 0 & \frac{z}{a} & \frac{1}{2} & 0 & 0 & \frac{1}{a} & 0 & -\frac{z}{a} & \frac{1}{2} & 0 \\
0 & 0 & -\frac{1}{a} & -\frac{y}{a} & 0 & \frac{1}{2} & 0 & 0 & \frac{1}{a} & \frac{y}{a} & 0 & \frac{1}{2}
\end{array}\right)
$$

And the strain matrix for the s-th rebar taking into account (5) has the following form - $\boldsymbol{\Phi}_{s}=\boldsymbol{\Phi}_{S}\left(x, y_{s}, z_{s}\right)$. Strains of the s-th rebar are expressed in terms of the nodal displacements as follows: $\vec{\varepsilon}_{s}=\boldsymbol{\Phi}_{s}\left(x, y_{s}, z_{s}\right) \mathbf{q}_{e}$, where $\vec{\varepsilon}_{s}$ is a vector composed of strain tensor components $\boldsymbol{\varepsilon}_{\mathrm{s}}$.

The above relations are substituted into (6) resulting in the finite element relationships - an expression for the vector of internal forces

$$
\mathbf{r}_{\text {int }}=\int_{V} \boldsymbol{\Phi}^{T}(x, y, z) \vec{\sigma} d V+\sum_{s} A_{s} \int_{0}^{a} \boldsymbol{\Phi}_{s}^{T}\left(x, y_{s}, z_{s}\right) \vec{\sigma}_{s} d x
$$

and for the consistent tangent stiffness matrix

$$
\mathbf{K}^{t}=\int_{V} \boldsymbol{\Phi}^{T}(x, y, z) \mathbf{C}^{t} \boldsymbol{\Phi}(x, y, z) d V+\sum_{s} A_{s} \int_{0}^{a} \boldsymbol{\Phi}_{s}^{T}\left(x, y_{s}, z_{s}\right) \mathbf{C}_{s}^{t} \mathbf{\Phi}_{s}\left(x, y_{s}, z_{s}\right) d x .
$$

Here $\vec{\sigma}, \vec{\sigma}_{s}$ are vectors composed of stress tensor components for concrete and reinforcement, respectively, $\mathbf{C}^{t}=\partial \vec{\sigma} / \partial \vec{\varepsilon}, \mathbf{C}_{s}^{t}=\partial \vec{\sigma}_{s} / \partial \vec{\varepsilon}_{s}$. Matrices $\mathbf{C}^{t}, \mathbf{C}_{s}^{t}$ are determined in accordance with the assumed constitutive model. The details are given in (Fialko, 2018).

\section{Stability of numerical behavior of the considered design model}

The presence of a descending branch of the $\sigma-\varepsilon$ diagram for concrete leads to the divergence of the numerical solution with mesh refinement after passing the yield point of the $\sigma-\varepsilon$ diagram (Kayhan, 2009; Pietruszczak \& Mroz, 1981) and others, (Figure 4, on the right). The reason for this behavior is that we can obtain any strain value $\varepsilon \in\left[\varepsilon_{\mathrm{u}}, \varepsilon_{\mathrm{s}}\right]$ on the descending branch at the given stress level $\sigma_{\mathrm{av}}$ (Figure 4, on the left). Different approaches have been proposed to overcome this ambiguity, for example (Bažant \& Pijaudier-Cabot, 1988), (e.g. Jouan et al., 2014) etc.

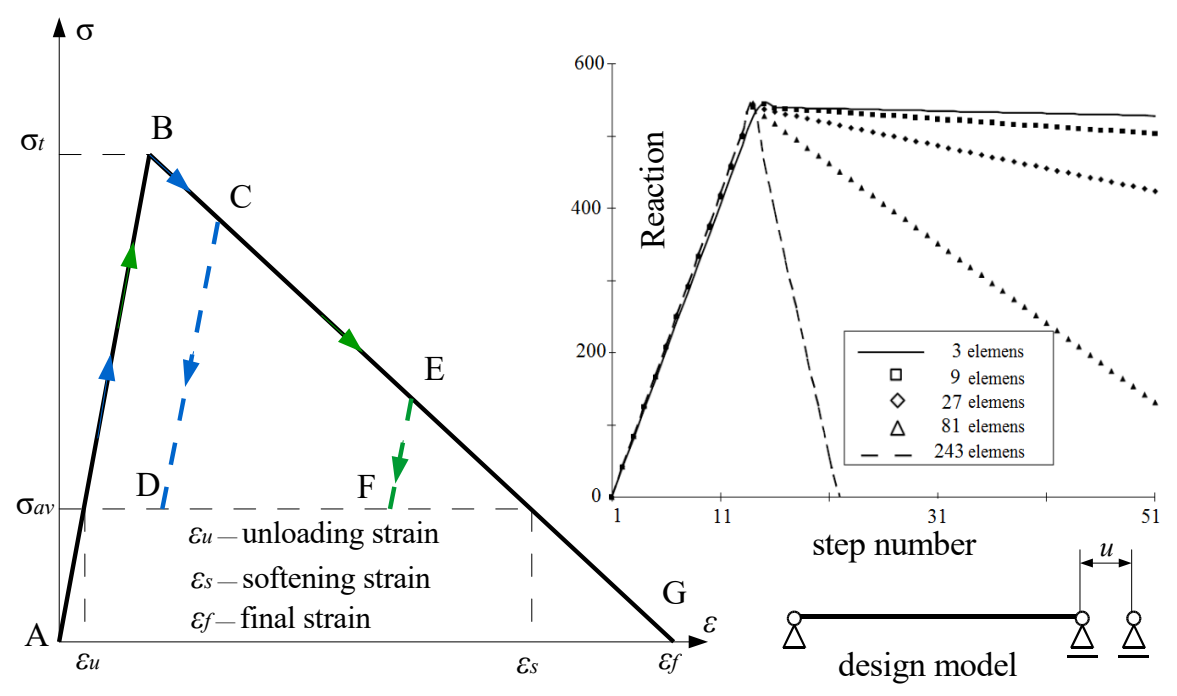

Figure 4. Divergence of the numerical solution with mesh refinement 
These approaches are quite complex and often require data on materials which are not readily available to the engineer. Therefore, a simple engineering idea is used in the proposed approach - the reinforcement should provide the regularity and stability of the numerical solution.

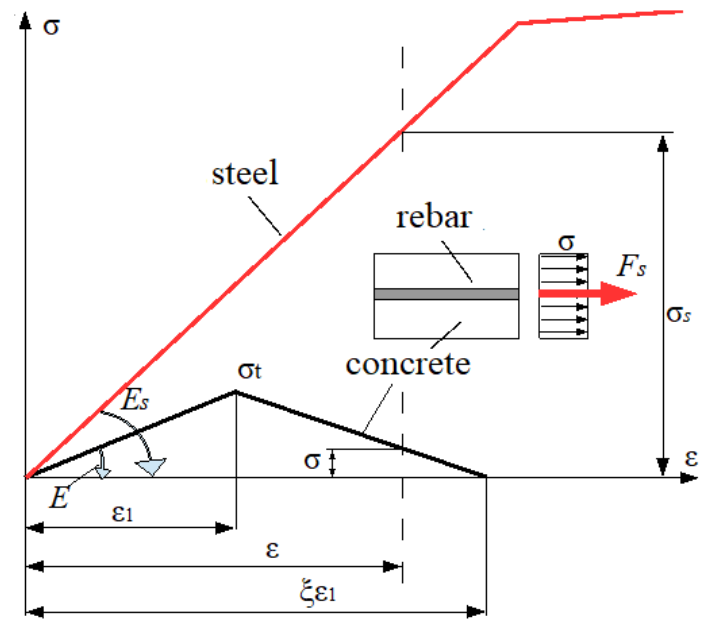

Figure 5. Mutual work of concrete and reinforcement in the tension area

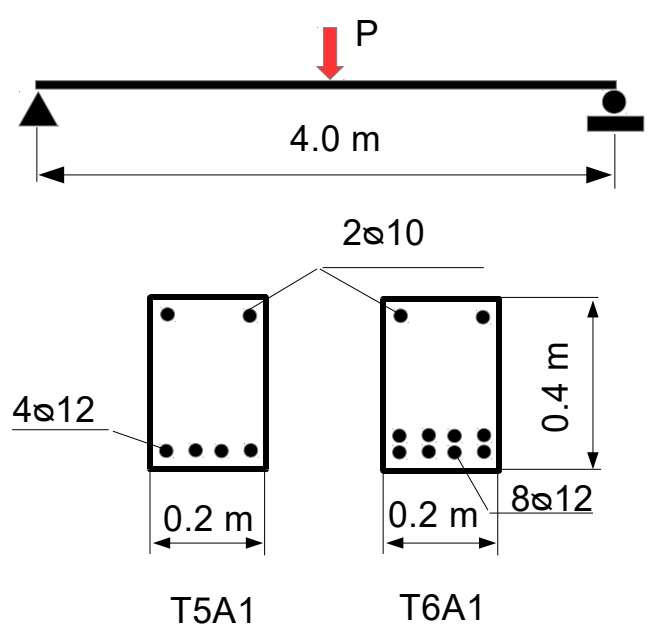

Figure 6. Three-point test. Design model and the reinforcement layout

Let's consider a reinforced concrete bar subjected to uniform tension (Figure 5). The longitudinal force in the bar is

$$
N(\varepsilon)=A \sigma+A_{s} \sigma_{s}=A E\left(\varepsilon_{1} \xi-\varepsilon\right) /(\xi-1)+A_{s} E_{s} \varepsilon, \quad \varepsilon=\varepsilon_{s}
$$

Here $A, A_{s}$ are the cross-sectional area of a bar and the cross-sectional area of a rebar, $\varepsilon_{1}=\sigma_{t} / E$, where $\varepsilon_{1}$ is the strain of concrete corresponding to $\sigma_{\mathrm{t}}, \mathrm{E}$ is the initial elastic modulus of concrete, $\xi$ is the parameter regulating the rate of decrease of stresses in concrete with increasing strain $\varepsilon$ within the descending branch, $E_{\mathrm{s}}$ is the elastic modulus of steel, $\varepsilon$ is the longitudinal strain. In order for a numerical solution to be stable, the function $\mathrm{N}(\varepsilon)$ has to be increasing for any value of $\varepsilon$. When $\varepsilon$ is in the range $\varepsilon \in\left[\varepsilon_{1}, \xi \varepsilon_{1}\right]$, it is true only when $\mathrm{dN}(\varepsilon) / \mathrm{d} \varepsilon>0$. This condition is satisfied automatically in other ranges until reinforcement works elastically. Differentiating (10) by $\varepsilon$, we obtain

$$
\xi>\xi_{\min }, \quad \xi_{\min }=1+\frac{1}{\mu_{s}} \frac{E}{E_{s}}, \quad \mu_{s}=\frac{A_{s}}{A} .
$$

Here $\mu_{\mathrm{s}}$ is the reinforcement ratio. This, in particular, implies that when there is no reinforcement $\left(\mu_{\mathrm{s}}=0\right) \xi_{\min } \rightarrow \infty$, the equilibrium state of the concrete bar within the descending branch will be unstable regardless of the $\xi$ value. In the case of a reinforced bar, the inequality (11) is a necessary condition for the stability of the numerical solution of a tensile reinforced concrete bar within the descending branch (Fialko \& Karpilovskyi, 2017).

In order to provide the stability of the numerical solution, it is also important to take into account the stiffness of reinforcement on transverse shear in addition to its axial stiffness. Otherwise, there will be zero rows and columns in the tangential stiffness matrix in the tension area of concrete, which leads to its singularity and to lack of convergence when Newton-Raphson method is used (Fialko, 2018).

\section{Numerical results}

In order to confirm the reliability of the proposed approach, the results obtained for a bent single-span beam (Figure 6) are compared with the results of the numerical solution (Belytschko \& Rabczuk, 2006), obtained by the particle method, and with the results of the experiment considered in the referenced paper. The following physical and mechanical characteristics are assumed for concrete: $E=28000 \mathrm{MPa}, \sigma_{c}=32 \mathrm{MPa}, \sigma_{t}=2.5 \mathrm{MPa}, v=0.22$, and for reinforcement $-E_{s}=200000 \mathrm{MPa}, \sigma_{y, s}=587 \mathrm{MPa}, E_{s}{ }^{\prime} / E_{s}=0.01$, where $E_{s}{ }^{\prime}$ and $\sigma_{y, s}$ are hardening modulus and yield strength of steel respectively. Longitudinal reinforcement resists tension, compression and shear. Due to symmetry, half of the beam is considered, which is evenly divided into 16 finite elements. The local axis OX of each finite element coincides with the axis of the beam, and the axis OZ is directed vertically upwards. Further mesh refinement does not lead to any noticeable changes in the numerical results. When the deformation theory of plasticity is used, it is assumed that $\xi=10$. For all constitutive models $\alpha=\beta=0$, and for the CM3 model $-\bar{\alpha}=0.54$ (the deviation of paraboloid from the circular shape is close to its maximum value). 


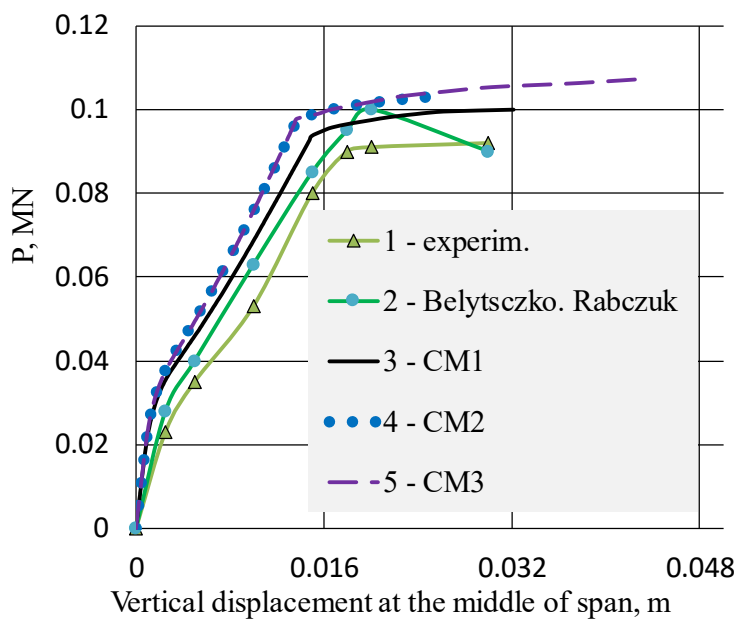

Figure 7. Load-displacement diagram for T5A1 specimen

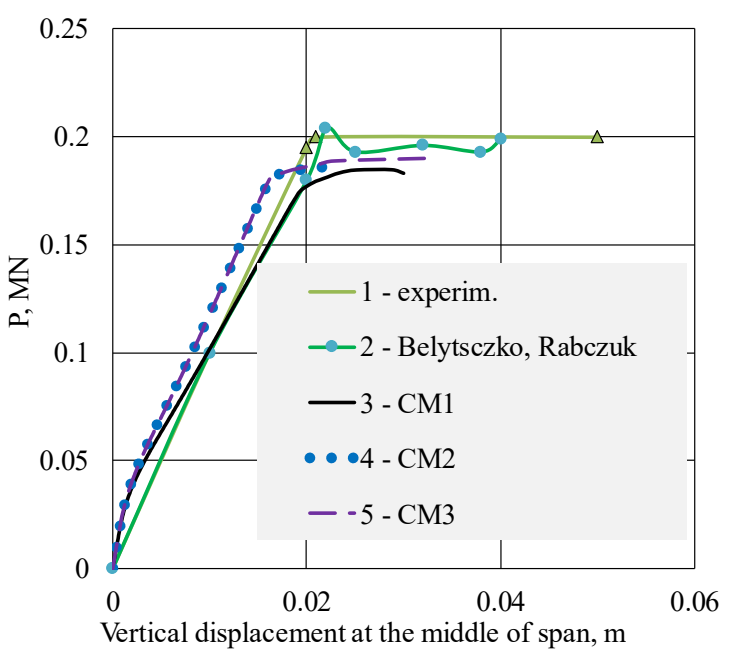

Figure 8. Load-displacement diagram for T6A1 specimen

Both the proposed approach and the particle method (Figures 7, 8) predict the load-bearing capacity of the considered bent beams with accuracy acceptable for practical calculations - within $10 \%$ compared with the results of the experiment. The behavior of the equilibrium state curves obtained by the above approaches also corresponds to the nature of the experimental results. However, the proposed approach is much simpler than the particle method. 96 nonlinear algebraic equations had to be solved in the proposed approach for the spatial problem. The plane stress state was considered in (Belytschko \& Rabczuk, 2006), 8200 particles were used in the design model, the number of equations is not specified. However, it is clear that the dimension of the design model of the particle method is much greater than the dimension of the design model of the proposed approach, while the accuracy of the results of both approaches in determining the load-bearing capacity is almost the same.

A design model of a multi-storey building from the SCAD Soft collection is shown in Figure 9. After performing the linear analysis and selecting the reinforcement, we have performed the elasto-plastic analysis of a fragment of the structure modeled by shell finite elements taking into account physical nonlinearity and reinforcement (Fialko \& Karpilovskyi, 2017; (Fialko, 2015, 2018), and also by the spatial frame finite elements considered in this paper. For floors, the direction of the reinforcing rods coincides with the axes of the global coordinate system OX and OY, and for bar elements - with the direction of the longitudinal axis of each bar. In the walls, reinforcing bars are arranged vertically and in the circumferential direction. For shell finite elements, the axis of the reinforcing bars can be rotated at an arbitrary angle relative to the local axis OX (Fialko, 2015, 2018).

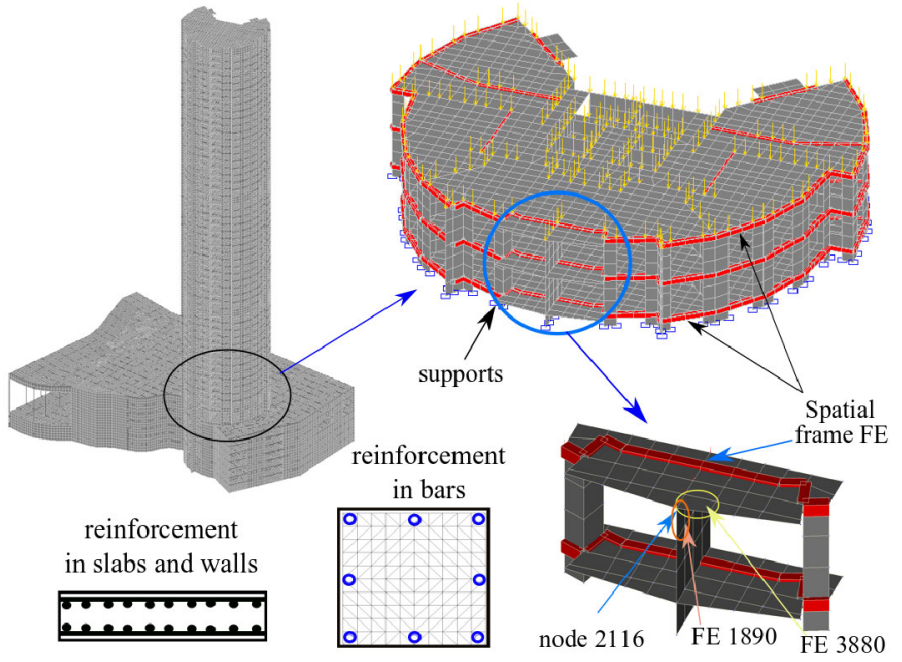

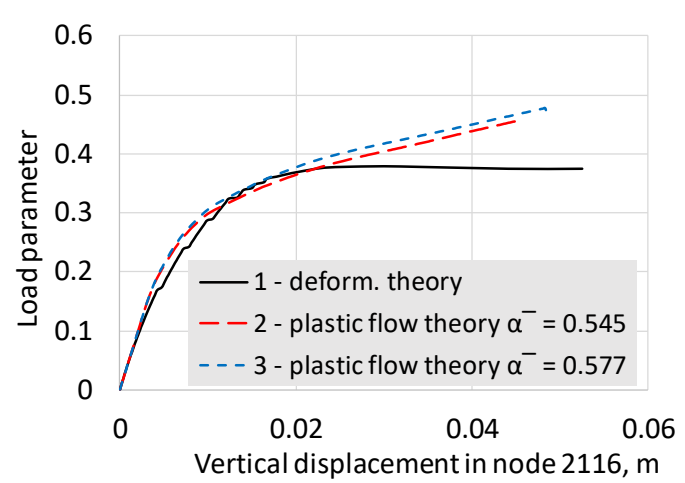

Figure 10. Load-displacement diagram

Figure 9. Design model of the fragment of a multi-storey building

The load from the overlying floors is applied to the upper edges of the walls of the design fragment, and a uniformly distributed constant load is applied to the floor slabs. All loads vary in proportion to one parameter. All elements have the same characteristics of concrete and steel: $E=34531 \mathrm{MPa}, v=0.2, \sigma_{\mathrm{c}}=25.5 \mathrm{MPa}, \sigma_{\mathrm{t}}=1.95 \mathrm{MPa}$, 
$E_{\mathrm{s}}=201000 \mathrm{MPa}, \sigma_{\mathrm{y}, \mathrm{s}}=408 \mathrm{MPa}, E_{\mathrm{s}}{ }^{\prime}=0.01 \mathrm{E}_{\mathrm{s}}$. The reinforcement ratio for walls is taken as $\mu_{\mathrm{x}}=\mu_{\mathrm{z}}=0.04$, for floor slabs $-\mu_{\mathrm{x}}=\mu_{\mathrm{y}}=0.006$, for beams and chords $-\mu=0.023$.

The load-displacement diagram for the control node of the design model is shown in Figure 10. Curve 1 corresponds to the deformation theory of plasticity, and curves 2 and 3 correspond to the theory of plastic flow. The yield criterion (e.g. Geniev et al., 1974) (CM3) was used for walls, and the Drucker-Prager criterion (CM2) was used for floor slabs. When $\alpha^{-}=0.577$ the yield surface is represented by a circular paraboloid, when $\alpha^{-}=0.545$ the deviation of the paraboloid from circular shape is close to its maximum value.

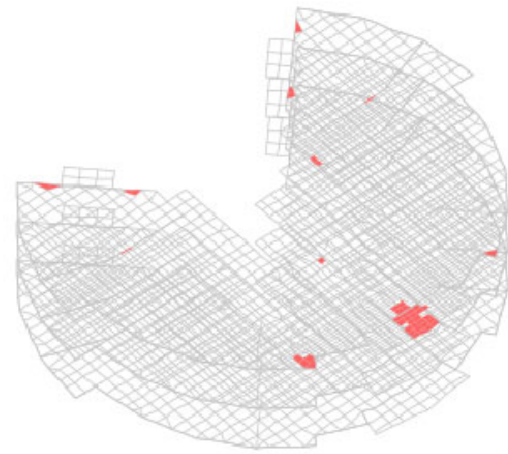

Limit state: rebar in floors exceeds the yield strength

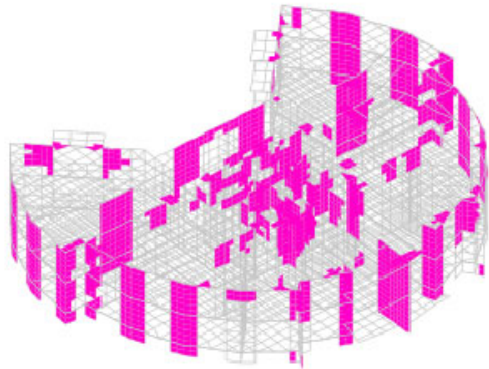

Limit state: vertical rebar exceeds the yield strength

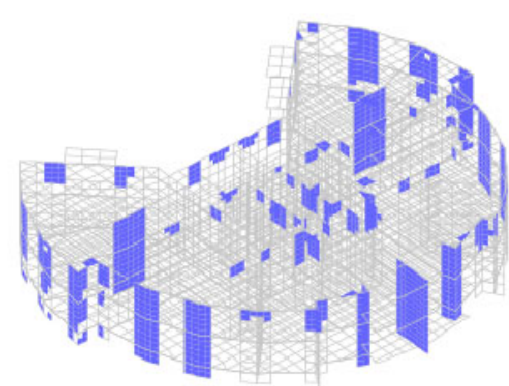

Limit state: concrete exceeds the compressive strength

Figure 11. Limit states of finite elements in the fragment

"Critical events" in the vicinity of the limit state are shown in Figure 11 for each shell finite element - the reinforcement in the tension area has reached the yield point, the reinforcement in the compression area has reached the yield point, the concrete in the compression area has exhausted its load-bearing capacity. Bar finite elements have not reached these limit states.

\section{Conclusions}

The proposed triangular and quadrilateral finite elements demonstrate an acceptable agreement with experimental results. It has been shown in the previous works. The spatial frame finite element shows an acceptable agreement with experimental results as well as with advanced numerical solutions obtained by other approaches. The uniform approach to both shell and spatial frame structural elements allows us to create the low-order finite elements for analysis of construction fragments consisting of thin-walled structural elements. It was found that fragments of floor slabs bounded by a closed contour formed by walls can work under conditions close to tension. Therefore, it is necessary in such cases to install strapping booms that reduce this tension effect.

\section{Acknowledgements}

We would like to express the deepest gratitude to our colleagues from SCAD Soft and to the users of our software for their comprehensive assistance, real design models, advice and suggestions.

\section{References}

ABAQUS 6.12 documentations. (2012). Retrieved from http://abaqus.software.polimi.it/v6.12/

ANSYS manuals. (1999). Retrieved from http://research.me.udel.edu/lwang/teaching/MEx81/ansys56manual.pdf

Bathe, K. J. (1996). Finite element procedures. New Jersey: Prentice Hall.

Bathe, K. J., Walczak, J., Welch, A., \& Mistry, N. (1989). Nonlinear analysis of concrete structures. Computers \& Structures, 32(3-4), 563-590. https://doi.org/10.1016/0045-7949(89)90347-7

Bažant, Z. P., \& Pijaudier-Cabot, G. (1988). Nonlocal continuum damage, localization instability and convergence. Journal of. Appl.ied Mech.anics, 55, (2), 287-293. https://doi.org/10.1115/1.3173674

Belytschko, T., \& Rabczuk, T. (2006). Application of particle methods to static fracture of reinforced concrete structures. International Journal of Fracture, 137(1-4), 19-49. https://doi.org/10.1007/s10704-005-3075-z

Bezukhov, N. I. (1961). Fundamentals of the theory of elasticity, plasticity and creep. Moscow: High school.

Criesfield, M. A. (2000). Non-linear finite element analysis for solids and structures (vol. 1: Essentials). New York: John Wiley \& Sons Ltd, Chichester. 
Criesfield, M. A. (2000). Non-linear finite element analysis for solids and structures (vol. 2: Advanced topics). New York: John Wiley \& Sons Ltd, Chichester.

DIANA manual. (2014). Retrieved from https://dianafea.com/manuals/d95/Diana.html)

Fialko, S. Yu. (2018). Application of finite element method to analysis of strength and bearing capacity of thin-walled concrete structures, taking into account the physical nonlinearity. Moscow: ASV, SCAD Soft.

Fialko, S. (2015). Quadrilateral finite element for analysis of reinforced concrete floor slabs and foundation plates. Applied Mechanics and Materials, 725-726, 820-835. https://doi.org/10.4028/www.scientific.net/AMM.725-726.820

Fialko, S., \& Karpilovskyi, V. (2017). Triangular and quadrilateral flat shell finite elements for nonlinear analysis of thin-walled reinforced concrete structures in SCAD software. In W. Pietraszkiewicz \& W. Witkowski (Eds.), Shell structures: theory and applications (vol. 4, chapter 83, pp. 367-370). CRC Press Taylor \& Francis Group, London, New York. https://doi.org/10.1201/9781315166605-83

Geniev, G. A., Kissyuk, V. N., \& Tyupin, G. A. (1974). The theory of plasticity of concrete and reinforced concrete. Moscow: Stroyizdat.

Ilyushin, A. A. (1948). Plasticity. Part one. Elasto-plastic deformations. Moscow-Leningrad: OGIZ.

Jirásek, M., \& Bažant, Z. P. (2002). Inelastic analysis of structures. WestSussex, England: John Wiley \& Sons, Ltd. Baffins Lane, Chichester.

Jouan, G., Kotronis, P., \& Collin, F. (2014) Using a second gradient model to simulate the behavior of concrete structural elements. Finite Elements in Analysis and Design, 90, 50-60. https://doi.org/10.1016/j.finel.2014.06.002

Karpilovskyi, V. S., Kryksunov, E. Z., Maliarenko, A. A., Fialko, S. Yu., Perelmuter, A. V., \& Perelmuter, M. A. (2018). SCAD Office.V.21. System SCAD++. Moscow: Publishing House SCAD SOFT. https://scadsoft.com/download/SCAD1033.pdf

Kayhan, E. (2009). Application of viscous and non-local integral type regularization schemes for softening plasticity (Master's thesis). Manufacturing Engineering Atilim University.

Pietruszczak, S., \& Mroz, Z. (1981). Finite element analysis of deformation of strain-softening materials. International Journal for Numerical Methods in Engineering, 17(3), 327-334. https://doi.org/10.1002/nme.1620170303

Sukumar, N., Moes, N., Moran, B., \& Belytschko, T. (2000). Extended finite element method for three-dimensional crack modelling. International Journal for Numerical Methods in Engineering, 48(11), 1549-1570. https://doi.org/10.1002/10970207(20000820)48:11<1549::AID-NME955>3.0.CO;2-A

Wang, L. C. (2013). Meso-scale numerical modeling of the mechanical behavior of reinforced concrete members. IACSIT International Journal of Engineering and Technology, 5(6), 680-684. http:॥doi: 10.7763/IJET.2013.V5.641 